\title{
Associations among eating behaviour traits, diet quality and food labelling: a mediation model
}

\author{
Raphaëlle Jacob ${ }^{1,2,3}$, Vicky Drapeau 2,3,4, Benoît Lamarche ${ }^{1,2}$, Éric Doucet ${ }^{5}$, \\ Sonia Pomerleau ${ }^{1,2}$ and Véronique Provencher ${ }^{1,2, *}$ \\ 'School of Nutrition, Laval University, Québec, Canada: ${ }^{2}$ Institute of Nutrition and Functional Foods (INAF), Laval \\ University, 2440 Boulevard Hochelaga, Québec, Canada, GIV 0A6: ${ }^{3}$ Quebec Heart and Lung Institute Research \\ Center, Laval University, Québec, Canada: ${ }^{4}$ Department of Physical Education, Laval University, Québec, Canada: \\ ${ }^{5}$ School of Human Kinetics, University of Ottawa, Ottawa, Canada
}

Submitted 17 September 2018: Final revision received 17 May 2019: Accepted 17 July 2019: First published online 26 November 2019

\begin{abstract}
Objective: To assess the associations among eating behaviour traits, food label use and diet quality and to evaluate if the association between eating behaviour traits and diet quality is mediated by food label use.

Design: Eating behaviour traits were assessed using the Three-Factor Eating Questionnaire (TFEQ), the Restraint Scale and the Intuitive Eating Scale, whereas food label use was measured with the Label Reading Survey. Diet quality (Canadian Healthy Eating Index) was assessed with an FFQ.

Setting: Cross-sectional study.

Participants: Adults ( $n$ 385; mean (SD): BMI $=26 \cdot 0(4 \cdot 9) \mathrm{kg} / \mathrm{m}^{2}$, age $=41 \cdot 1(15 \cdot 0)$ years) involved in two previous experimental studies.

Results: When controlling for potential covariates, general food label use ( $\beta=1 \cdot 18$ (se $0 \cdot 26$ ), $P<0 \cdot 0001$ ) was the main determinant of diet quality, explaining $6.7 \%$ of its variance. General food label use partly mediated the association between TFEQ-cognitive restraint and diet quality; the indirect effect $\left(\beta_{\text {indirect }}(\mathrm{SE})\right.$; $95 \% \mathrm{CI})$ was stronger in men $(0.32(0 \cdot 10) ; 0.15,0.55)$ than women $(0 \cdot 16(0.05)$; $0 \cdot 08,0 \cdot 27)$. General food label use also partly mediated the negative association between unconditional permission to eat and diet quality; the indirect effect $\left(\beta_{\text {indirect }}(\mathrm{SE}) ; 95 \% \mathrm{CI}\right)$ was also stronger in men $(-1.88(0.55) ;-3.11,-0.96)$ than women $(-1.03(0.33) ;-1.81,-0.49)$.

Conclusions: General food label use was the main determinant of diet quality and partly mediated the association between eating behaviour traits and diet quality. The stronger mediating effect observed in men suggests they rely more on food labelling when attempting to restrained themselves, which translates into better diet quality.
\end{abstract}

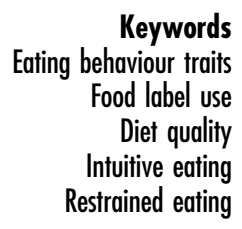

Many factors are involved in the aetiology of obesity, including behavioural and psychological factors. Among these, eating behaviour traits that have been widely studied in association with body weight are cognitive restraint, disinhibition and susceptibility to hunger ${ }^{(1,2)}$. Dietary habits are also involved in weight management. Improvements in diet quality have indeed been associated with a lower weight gain over a 20 -year period ${ }^{(3)}$. One way that eating behaviours can influence body weight is through diet quality, which can impact energy intake. Accordingly, cognitive restraint, defined as the intent to restrain food intake in order to control body weight, has been associated with higher intake of healthy foods such as green vegetables ${ }^{(4)}$. Moreover, flexible control, a more relaxed or graduated approach towards eating, dieting and weight, has been associated with better diet quality ${ }^{(5)}$. Disinhibition, defined by a loss of control over eating, has been associated with higher intake of energy-dense foods ${ }^{(2)}$. Susceptibility to hunger, which refers to the susceptibility to feel hungry triggered by internal or external cues, is strongly associated with disinhibition $^{(6,7)}$ and has been positively associated with energy intake ${ }^{(8)}$. Intuitive eating, an eating style that relies on hunger and satiety cues to determine when, what and how much to eat ${ }^{(9)}$, showed a very weak but positive association with vegetable intake ${ }^{(10)}$ and a weak and positive association with self-reported food diversity ${ }^{(11)}$, although research regarding this eating behaviour is more limited and no association with dietary intake has also been 
reported $^{(12)}$. Moreover, gender differences have been observed in these eating behaviour traits. Women generally have higher levels of cognitive restraint and disinhibition than men ${ }^{(13-15)}$. Gender difference in susceptibility to hunger is less clear, as studies observed either no difference between men and women ${ }^{(13,15)}$ or that women present a lower ${ }^{(14)}$ level of susceptibility to hunger than men. Finally, a higher level of intuitive eating has been observed in men compared with women ${ }^{(11,16,17)}$.

In addition to eating behaviour traits, food labelling, which represents a primary source of nutrition information, may be another factor influencing diet quality. Accordingly, food labelling has been proposed as a tool to help individuals make better and informed food choices ${ }^{(18)}$ and it has been reported that food label use is associated with better diet quality ${ }^{(19,20)}$. Studies generally show that women report using food labels more frequently than men ${ }^{(20,21)}$ and they are more likely to report that food labels influence their food choices ${ }^{(20)}$. Despite this beneficial effect of food labels on food choices and diet quality, several studies have also shown that food labelling may be confusing for some individuals ${ }^{(18,20)}$ and their use does not always translate into healthier food choices or eating habits ${ }^{(22)}$. These conflicting results may be explained by different uses of food labelling among individuals presenting diverse eating behaviour traits. For instance, restrained individuals may be more receptive to food labels, since nutrition information could be viewed for them as salient cues to support dieting rules, which may not always be in accordance to healthy eating patterns ${ }^{(22)}$. Indeed, a greater use of the nutrition facts table has been associated with an increased likelihood of engaging in both healthy and unhealthy weight-control behaviours $^{(23)}$ and individuals attempting to control their body weight have also reported a greater use of food labels ${ }^{(20,21)}$. Consistent with this previous result, Christoph et al. recently showed that while nutrition facts use was unrelated to intuitive eating among young women, it was associated with a lower level of intuitive eating in young men ${ }^{(23)}$. That study also observed that greater nutrition facts use in women was associated with a greater likelihood of engaging in binge eating ${ }^{(23)}$, an eating disorder that has been positively associated with disinhibition ${ }^{(2)}$. To our knowledge, Christoph et al.'s was the first study to specifically assess the association between one of the specific eating behaviour traits presented above, namely intuitive eating, and the frequency of food label use $\mathrm{e}^{(23)}$ and no study has yet assessed the associations between the other eating behaviour traits presented above and food label use. While the need to better understand how individuals who may be at risk for disordered eating use food labels was recently emphasized ${ }^{(23)}$, no study has yet examined the associations among eating behaviour traits, food label use and diet quality in a mediation model which allows to identify the indirect effect by which eating behaviour traits are associated with diet quality.
The primary aim of the present study was thus to assess the associations among eating behaviour traits (i.e. cognitive restraint, disinhibition, susceptibility to hunger and intuitive eating), food label use and diet quality in men and women. A second aim was to evaluate if the associations between eating behaviour traits and diet quality are mediated by food label use. Based on the previous but limited literature, three hypotheses were stated: (i) cognitive restraint and intuitive eating are positively associated with diet quality and conversely disinhibition is negatively associated with diet quality, while susceptibility to hunger is not associated with diet quality; (ii) cognitive restraint, disinhibition and susceptibility to hunger are positively associated with food label use, whereas intuitive eating shows a negative association with food label use; and (iii) the use of food labels may partly mediate the association between cognitive restraint and diet quality. The first hypothesis is confirmatory but is a previous step for the other two hypotheses which are exploratory, except for the associations of intuitive eating and disinhibition with food label use, since the association has been previously observed or a similar behaviour has been associated with food label use, respectively.

\section{Methods}

\section{Participants}

The current cross-sectional study was conducted among participants resulting from a posteriori pooling of participants involved in two previous studies ${ }^{(24,25)}$. These studies aimed to assess the impact of food labelling on energy intake, appetite sensations and food perceptions during either a $10 \mathrm{~d}$ experimental period, where they received three ad libitum take-home meals per day ${ }^{(24)}$, or a single ad libitum snack test ${ }^{(25)}$. In the $10 \mathrm{~d}$ experimental period, a label on the lunch meal indicating either 'low-fat' or the energy content of the meal, or no label as a control, differentiated the three experimental groups ${ }^{(24)}$. In the snack test, oatmeal-raisin cookies were described as healthy (i.e. high-fibre oatmeal), diet (i.e. satiating effect) or hedonic (less healthy ingredients, i.e. brown sugar and butter), depending on the experimental group ${ }^{(25)}$. Note that the experimental conditions of these two studies had no impact on measured energy intake ${ }^{(24,25)}$. Participants were recruited through different media at Laval University or in the Quebec City area. Inclusion criteria for the present study were as follows: age between 18 and 68 years; self-reported stable body weight $( \pm 2.5 \mathrm{~kg})$ in the last 2 to 3 months prior to the study; no medications that could interfere with study outcomes (e.g. corticosteroids, antidepressants, antipsychotics); no weight-related or chronic health diseases (e.g. eating disorders, type 1 or type 2 diabetes, uncontrolled hypo- or hyperthyroidism, food allergies); and not being pregnant or lactating. Participants were blinded to the objectives of each study. 
Table 1 Summary of measurement times (before or after experimentation) of the two previous experimental studies

\begin{tabular}{|c|c|c|c|c|}
\hline \multirow[b]{2}{*}{ Measure } & \multicolumn{2}{|c|}{$\begin{array}{l}\text { Carbonneau } \\
\text { et al. } .^{(24)}\end{array}$} & \multicolumn{2}{|c|}{ Gravel et al. ${ }^{(25)}$} \\
\hline & Before & After & Before & After \\
\hline Height & $x$ & & & $x$ \\
\hline Weight & $x$ & & & $x$ \\
\hline $\begin{array}{l}\text { FFQ } \\
\text { Questionnaires }\end{array}$ & $x$ & & & $x$ \\
\hline $\begin{array}{c}\text { Three-Factor Eating } \\
\text { Questionnaire }\end{array}$ & & $x$ & & $x$ \\
\hline Restraint Scale & & $x$ & & $x$ \\
\hline Intuitive Eating Scale & & $x$ & & $x$ \\
\hline Food label use & $\mathrm{x}$ & & & $\mathrm{x}$ \\
\hline Sociodemographic & & $\mathrm{x}$ & & $\mathrm{x}$ \\
\hline
\end{tabular}

\section{Measurements}

\section{Anthropometric measurements}

Height was measured to the nearest $0 \cdot 1 \mathrm{~cm}$ using a standard stadiometer and body weight was measured to the nearest $0.1 \mathrm{~kg}$ with a digital scale. BMI was calculated as body weight divided by height squared $\left(\mathrm{kg} / \mathrm{m}^{2}\right)$. Table 1 presents a summary of measurement times of the previous studies.

\section{Diet quality assessment}

Self-reported dietary intake was measured using a validated FFQ, either in interview $(n 269)^{(26)}$ or using a web-based self-administered format $(n 116)^{(27)}$. The webbased FFQ contained 136 items and was developed based on the interviewer-administered FFQ which contained ninety-one items with a total of thirty-three sub-questions. Both FFQ measure dietary intakes over the last month. The web-based FFQ required approximately $45 \mathrm{~min}$ to complete and the interviewer-administered FFQ required between 30 and $45 \mathrm{~min}$. The nutritional analysis was based on the Nutrition Data System for Research, version 4.03, for the interviewer-administered FFQ and on a food composition database created based on the Nutrition Data System for Research, version 4.03, and the Canadian Nutrient File, version 2007b, for the web-based FFQ. Servings of the 2007 Canada's Food Guide were computed using an Excel file (Microsoft Office 2007) created for that purpose or electronically, depending on the FFQ. A reasonable agreement, as assessed by cross-classification between quartiles of dietary intake, has been demonstrated (i.e. a mean (SD) of 84.3 (5.9) \% of participants classified within the same or adjacent quartiles of dietary intakes for all nutrients, with a mean (SD) of $2.5(2.0) \%$ classified in non-adjacent quartiles), and significant correlations for the majority of nutrients (average of Pearson correlation coefficients (SD), $r=0.59(0 \cdot 15))$ have also been shown between both $\mathrm{FFQ}^{(27)}$. Diet quality was assessed using the Healthy Eating Index (HEI) adapted for the Canadian nutrition recommendations ${ }^{(28)}$. This index reflects the global quality of the diet on a 100-point score comprising ten components. The HEI score was calculated based on data obtained from the nutritional analysis.

\section{Questionnaires}

Eating behaviour traits were assessed using a validated French version ${ }^{(29)}$ of the Three-Factor Eating Questionnaire (TFEQ) ${ }^{(1,15)}$, the Restraint Scale ${ }^{(30)}$ and the Intuitive Eating Scale ${ }^{(31)}$ translated in French. The TFEQ measures cognitive restraint (twenty-one items, Cronbach's $\alpha=0.81$ ) and its two subscales, i.e. rigid and flexible control (seven items each, Cronbach's $\alpha=0.59$ and 0.62 , respectively), disinhibition (sixteen items, Cronbach's $\alpha=0.71$ ) and susceptibility to hunger (fourteen items, Cronbach's $\alpha=0.72$ ). The Restraint Scale (ten items, Cronbach's $\alpha=0.64$ ) also assesses restrained eating but combined with a weight fluctuation factor ${ }^{(30)}$. The Intuitive Eating Scale measures total intuitive eating score (twenty-one items, Cronbach's $\alpha=0.85$ ) as well as three subscales, namely unconditional permission to eat (nine items), eating for physical rather than emotional reasons (six items) and reliance on internal hunger and satiety cues to determine when and how much to eat (six items) (Cronbach's $\alpha=0.79,0.89$ and 0.74 , respectively) ${ }^{(31)}$.

A French version of the validated Label Reading Survey ${ }^{(32)}$ was used to measure a general (i.e. general food label use) and specific behaviour (i.e. item seeking on food labels) towards food label use. The Cronbach's $\alpha$ coefficient for the whole questionnaire, that also measures attitudes and knowledge towards food labels, was $0 \cdot 78$, which was similar to the value of the original questionnaire (i.e. $0 \cdot 80)^{(32)}$. Cronbach's $\alpha$ coefficients were 0.57 and 0.81 for general food label use and item seeking on food labels, respectively. General food label use is measured as the sum of three items on a 5 -point scale $(1=$ 'never' to $5=$ 'always'). For example, the following item was used: "When you purchase a food product for the first time, do you look at the Nutrition Facts label on the package?'. Item seeking is measured as the sum of fifteen items appearing on the Nutrition Facts table (e.g. serving size, calories, sodium, etc.) and two items related to health and nutrition claims on food labels. Participants were asked to indicate whether they used each item when looking at food labels ( $1=$ 'no'; $2=$ 'yes'). This French version of the Label Reading Survey was adapted to the Canadian food labelling context (e.g. by replacing the word 'Americans' for 'Canadians' and modifying examples of American Nutrition Facts labels for Canadian labels), but these adaptations did not change the nature of the questionnaire. No changes were made to the items related to general food label use and the only change to item seeking on food labels related items was modifying 'Calories from fat' for 'Percentage of daily value from fat' as the former do not appear on the Canadian Nutrition Facts Table. Participants also completed a sociodemographic questionnaire.

\section{Statistical analyses}

Descriptive statistics (means and their standard deviations, or frequencies) were computed to assess participant characteristics, eating behaviour traits and food label use in the 
whole sample. The $t$ test and $\chi^{2}$ analyses were performed to assess differences between men and women. To account for the possibility of under- and over-reporting of dietary intakes, participants having a ratio of self-reported energy intake to estimated BMR, calculated with the HarrisBenedict equation, lower than 1.14 and higher than 2.40 were excluded from the analyses ${ }^{(33)}$. A ratio below $1 \cdot 14$ rather than 1.35 was chosen to identify under-reporters of energy intake as it represents the lowest energy intake to BMR ratio that may reflect actual energy intake over a given period of time ${ }^{(34)}$. Moreover, this ratio was chosen because restrained eaters and individuals with obesity are more likely to under-report dietary intake $^{(33,35)}$ and excluding these individuals was not desired given the objectives of the study. A total of eighty-eight and thirty-five participants were identified as under- and over-reporters, respectively. Therefore, 385 participants were included in the analyses. Analyses were adjusted for the experimental conditions of the two previous studies by creating five indicator variables (i.e. experimental groups 1 to 3 were assigned to the three groups of Carbonneau et al.'s study ${ }^{(24)}$ and experimental groups 4 to 6 were assigned to the three groups of Gravel et al.'s study ${ }^{(25)}$. The indicator variables were created for experimental conditions 1, 2, 4, 5 and 6, and the control group of Carbonneau et al.'s study ${ }^{(24)}$ (experimental condition 3) was used as the reference). These indicator variables were added as covariates in each analysis, even though no difference was observed in the main eating behaviour traits and food label use among the different experimental groups of the two previous studies (data not shown, $P>0.05$ ).

Partial Pearson's correlations were used to assess the associations among eating behaviour traits, diet quality and food label use variables. These associations were first tested in a model that was adjusted only for experimental conditions and then in a model that was further adjusted for potential confounders (i.e. experimental conditions, age, gender, BMI, education level and household income $)^{(5,20)}$. Age, BMI, gender, education level (2 to 5; no participant reported having no education level or not having completed elementary school, which was coded as 1) and household income ( 1 to 6 ) were treated as continuous variables while gender $(0=$ men; $1=$ women $)$ was treated as a binary variable. Total scores of the main eating behaviour traits and food label use variables that were significantly associated with HEI score were included in multiple stepwise regression analyses. These analyses were performed using an unadjusted model except for experimental conditions, and a fully adjusted model considering experimental conditions, BMI, age, gender, education level and household income as covariates. A second series of multiple stepwise regressions was run using the subscales of eating behaviour traits and food label use variables that were significantly associated with HEI score, again in an unadjusted model, except for experimental conditions, and a fully adjusted model for potential confounders.

Moderated mediation analyses were conducted to assess whether food label use mediates the association between eating behaviour traits and diet quality, and whether the mediation effect varies according to gender since gender differences have been observed in eating behaviour traits $^{(11,13-17)}$, food label use ${ }^{(20)}$ and diet quality ${ }^{(5)}$. These analyses were conducted with the use of model 58 in the Process macro version 2.16.3 for SAS that calculates bias-corrected $95 \%$ CI using bootstrapping with 5000 samples $^{(36)}$. Based on the location of the gender interaction identified, the analysis was rerun using the most suitable model (i.e. model 14 or 7 ) and if no moderated mediation was observed, the simple mediation model was used (i.e. model 4). The mediations were tested only in the model that was fully adjusted for covariates while considering gender as a potential moderator rather than a covariate. In cases where no moderating effect was observed, gender was thereafter considered as a covariate. Statistical significance was set to $P<0.05$. Bonferroni adjustments for multiple comparisons were not used because of the exploratory nature of the present study ${ }^{(37)}$, particularly regarding mediation analyses among eating behaviour traits, diet quality and food label use, since correlation analyses are generally a previous step for mediation analyses. It is however possible that chance associations are presented for some findings, especially for those close to a $P$ value of $0 \cdot 05$. However, to minimize this possibility, the only mediation models tested were those where significant associations between the independent and dependent variables (path $c$ ), between the independent variable and the mediator (path $a$ ) and between the mediator and the dependent variable (path $b$ ) were observed, according to the traditional view of interpretation of mediation analysis according to Baron and Kenny ${ }^{(38)}$. This rationale was used because the present study's aim was to better understand the observed associations between eating behaviour traits and diet quality. All statistical analyses were performed using the statistical software package SAS version 9.4.

\section{Results}

\section{Participant characteristics}

Mean (SD) age of participants (women, $n$ 265; men, $n$ 120) was $42.9(15.1)$ and $37.0(14 \cdot 1)$ years for women and men, respectively, and slightly more than two-thirds of the sample were women (Table 2). Women were significantly older and had a higher BMI, HEI score and level of restrained eating, as assessed with the TFEQ or with the Restraint Scale, compared with men. Women also presented a higher level of disinhibition and a lower intuitive eating score than men (Table 2). 
Table 2 Participant characteristics, eating behaviour traits, diet quality and food label use, of the whole sample and by gender, among women and men involved in two previous experimental studies ${ }^{(24,25)}$

\begin{tabular}{|c|c|c|c|c|c|c|c|}
\hline \multirow[b]{2}{*}{ Variable } & \multicolumn{2}{|c|}{ Total $(n 385)$} & \multicolumn{2}{|c|}{ Women (n265) } & \multicolumn{2}{|c|}{ Men $(n 120)$} & \multirow[b]{2}{*}{$P^{*}$} \\
\hline & Mean or \% & SD & Mean or $\%$ & SD & Mean or \% & SD & \\
\hline Gender (\%) & - & - & $68 \cdot 8$ & - & $31 \cdot 2$ & - & $<0.0001$ \\
\hline Age (years) & $41 \cdot 1$ & $15 \cdot 0$ & $42 \cdot 9$ & $15 \cdot 1$ & $37 \cdot 0$ & $14 \cdot 1$ & 0.0004 \\
\hline BMI $\left(\mathrm{kg} / \mathrm{m}^{2}\right)$ & $26 \cdot 0$ & 4.9 & $26 \cdot 4$ & 5.5 & $25 \cdot 1$ & 3.3 & 0.006 \\
\hline Overweight/obese (\%) & $50 \cdot 4$ & - & $49 \cdot 8$ & - & 51.7 & - & 0.74 \\
\hline \multicolumn{8}{|l|}{ Education level $(\%) \dagger$} \\
\hline Elementary school & 0.8 & - & $1 \cdot 2$ & - & 0.0 & - & $0.34 \uparrow$ \\
\hline High school & $12 \cdot 5$ & - & 11.7 & - & 14.4 & - & \\
\hline College & $30 \cdot 7$ & - & 28.4 & - & $35 \cdot 6$ & - & \\
\hline University & $55 \cdot 7$ & - & 58.4 & - & $50 \cdot 0$ & - & \\
\hline Prefer not to answer & 0.3 & - & 0.4 & - & 0.0 & - & \\
\hline \multicolumn{8}{|l|}{ Household income (\$CAN; \%) $\ddagger$} \\
\hline$<20000$ & $21 \cdot 3$ & - & $19 \cdot 7$ & - & 24.8 & - & 0.71 \\
\hline $20000-39999$ & $17 \cdot 8$ & - & $17 \cdot 3$ & - & $18 \cdot 8$ & - & \\
\hline $40000-59999$ & $20 \cdot 0$ & - & 20.5 & - & $18 \cdot 8$ & - & \\
\hline $60000-79999$ & $14 \cdot 6$ & - & $13 \cdot 8$ & - & $16 \cdot 2$ & - & \\
\hline $80000-99999$ & $7 \cdot 3$ & - & $8 \cdot 3$ & - & $5 \cdot 1$ & - & \\
\hline$\geq 100000$ & 11.9 & - & $12 \cdot 2$ & - & $11 \cdot 1$ & - & \\
\hline Prefer not to answer & $7 \cdot 3$ & - & $8 \cdot 3$ & - & $5 \cdot 1$ & - & \\
\hline HEI score (scale 0 to 100 ) & $79 \cdot 3$ & $11 \cdot 0$ & $80 \cdot 5$ & $10 \cdot 5$ & $76 \cdot 6$ & 11.5 & 0.001 \\
\hline \multicolumn{8}{|l|}{ Eating behaviours } \\
\hline Cognitive restraint (scale 1 to 21 ) & $7 \cdot 6$ & 4.3 & $8 \cdot 2$ & $7 \cdot 7$ & $6 \cdot 2$ & 3.8 & $<0.0001$ \\
\hline Rigid control (scale 1 to 7 ) & $2 \cdot 1$ & 1.6 & $2 \cdot 4$ & $1 \cdot 7$ & $1 . \overline{6}$ & 1.3 & $<0.0001$ \\
\hline Flexible control (scale 1 to 7 ) & $2 \cdot 8$ & 1.7 & 3.0 & $1 \cdot 7$ & 2.4 & 1.5 & 0.002 \\
\hline Disinhibition (scale 1 to 16 ) & 5.5 & 2.9 & $5 \cdot 8$ & 3.0 & 4.8 & $2 \cdot 6$ & 0.002 \\
\hline Susceptibility to hunger (scale 1 to 14 ) & 4.5 & $3 \cdot 0$ & 4.7 & $3 \cdot 0$ & 4.3 & $3 \cdot 1$ & 0.27 \\
\hline Restraint (scale 0 to 35$) \|$ & $13 \cdot 2$ & 4.7 & 13.9 & 4.6 & 11.6 & 4.6 & $<0.0001$ \\
\hline Intuitive eating (scale 1 to 5 ) & 3.4 & 0.5 & $3 \cdot 3$ & 0.5 & $3 \cdot 6$ & 0.5 & $<0.0001$ \\
\hline Unconditional permission to eat (scale 1 to 5 ) & $3 \cdot 2$ & 0.7 & $3 \cdot 1$ & 0.6 & 3.4 & 0.7 & $<0.0001$ \\
\hline Eating for physical rather than emotional reasons (scale 1 to 5 ) & 3.4 & 0.9 & $3 \cdot 2$ & 0.9 & 3.8 & 0.9 & $<0.0001$ \\
\hline Reliance on internal hunger and satiety cues (scale 1 to 5 ) & $3 \cdot 6$ & 0.6 & 3.6 & 0.6 & 3.6 & 0.5 & 0.42 \\
\hline General food label use (scale 3 to 15 ) & $9 \cdot 7$ & $2 \cdot 3$ & $9 \cdot 8$ & $2 \cdot 2$ & 9.4 & 2.5 & 0.07 \\
\hline Item seeking on food labels (scale 17 to 34 ) & $26 \cdot 7$ & $3 \cdot 8$ & $27 \cdot 0$ & 3.5 & $26 \cdot 2$ & 4.3 & 0.08 \\
\hline
\end{tabular}

Values are presented as means and standard deviations for continuous variables, or as frequencies for categorical variables.

$\mathrm{HEI}$, Canadian Healthy Eating Index (28).

${ }^{*} P$ values indicate gender differences.

†Missing values, $n 10$ (women, $n 8$; men, $n 2$ ).

‡Missing values, $n 14$ (women, $n 11$; men, $n 3$ ).

§Assessed with the Three-Factor Eating Questionnaire ${ }^{(1,29)}$

$\|$ Assessed with the Restraint Scale ${ }^{(30)}$.

ףP value from Fisher's exact test.

\section{Associations of eating behaviour traits and food label use with diet quality}

TFEQ-cognitive restraint and its subscales were positively associated with HEI score in the model adjusted only for experimental conditions and in the fully adjusted model (Table 3). Intuitive eating was negatively, but weakly, associated with HEI score in the model adjusted for experimental conditions $(P=0.03)$ whereas the association was no longer significant in the fully adjusted model $(P=0.052)$. A negative association was observed with one of the intuitive eating subscales, namely unconditional permission to eat, and diet quality in both models. Scores reflecting general food label use and item seeking on food labels were all positively associated with HEI score in each model (Table 3).

\section{Associations among eating behaviour traits and food label use}

Positive correlations were observed for the associations of TFEQ-cognitive restraint, rigid control and flexible control with general food label use and item seeking on food labels (Table 4). Small but positive correlations were also observed for disinhibition and susceptibility to hunger with general food label use in the fully adjusted model. Restrained eating, assessed with the Restraint Scale, was positively associated with general food label use but not with item seeking on food labels. Intuitive eating and its subscale unconditional permission to eat were negatively associated with general food label use and with item seeking on food labels in both statistical models, while the subscale eating for physical rather than emotional reasons was negatively associated with general food label use in the fully adjusted model (Table 4).

\section{Multiple regression analyses}

The first multiple regression model tested for diet quality (HEI score) included TFEQ-cognitive restraint, intuitive eating, general food label use, item seeking on food labels as 
Table 3 Associations of eating behaviour traits and food label use with diet quality (HEI score) among women and men involved in two previous experimental studies ${ }^{(24,25)}$

\begin{tabular}{|c|c|c|c|c|}
\hline \multirow[b]{2}{*}{ Variable } & \multicolumn{2}{|c|}{$\begin{array}{l}\text { Unadjusted } \\
\text { model }\end{array}$} & \multicolumn{2}{|c|}{$\begin{array}{l}\text { Fully adjusted } \\
\text { model }\end{array}$} \\
\hline & $r$ & $P$ & $r$ & $P$ \\
\hline Cognitive restraint ${ }^{\star}$ & 0.26 & $<0.0001$ & 0.20 & 0.0004 \\
\hline Rigid control & 0.19 & 0.0003 & 0.13 & 0.02 \\
\hline Flexible control & 0.24 & $<0.0001$ & 0.17 & 0.003 \\
\hline Disinhibition & -0.05 & 0.31 & -0.03 & 0.59 \\
\hline Susceptibility to & 0.01 & 0.90 & 0.06 & 0.32 \\
\hline Restraint $†$ & 0.03 & 0.62 & 0.00 & 0.98 \\
\hline Intuitive eating & -0.11 & 0.03 & -0.11 & 0.052 \\
\hline $\begin{array}{l}\text { Unconditional permission } \\
\text { to eat }\end{array}$ & $-0 \cdot 20$ & 0.0001 & $-0 \cdot 16$ & 0.003 \\
\hline $\begin{array}{l}\text { Eating for physical rather } \\
\text { than emotional reasons }\end{array}$ & 0.02 & 0.72 & 0.03 & 0.55 \\
\hline $\begin{array}{l}\text { Reliance on internal hunger } \\
\text { and satiety cues }\end{array}$ & -0.03 & 0.57 & $-0 \cdot 10$ & 0.07 \\
\hline General food label use & 0.30 & $<0.0001$ & 0.27 & $<0.0001$ \\
\hline Item seeking on food labels & 0.20 & 0.0002 & 0.19 & 0.00 \\
\hline
\end{tabular}

HEI, Canadian Healthy Eating Index ${ }^{(28)} ; r$, partial Pearson's correlation coefficient Unadjusted model (n 349 to 384 ): adjusted only for experimental conditions.

Fully adjusted model ( $n 317$ to 342 ): adjusted for experimental conditions, age, gender, BMI, education level and household income. Prefer not to answer for education level and household income were recoded as missing data. Education level and household income were treated as continuous variables. Five indicator variables were created for experimental conditions and the control group of Carbonneau et al.'s study ${ }^{(24)}$ was used as reference.

${ }^{*}$ Assessed with the Three-Factor Eating Questionnaire ${ }^{(1,29)}$.

†Assessed with the Restraint Scale ${ }^{(30)}$.

well as experimental conditions. General food label use $(\beta=1.21$ (se 0.26), $P<0.0001)$ and TFEQ-cognitive restraint $(\beta=0.39$ (se 0.15), $P=0.009)$ explained 6.4 and $2 \cdot 1 \%$ of the variance in the HEI score, respectively. The model explained $11.9 \%$ of the variance in the HEI score $(P<0.0001)$. Adding potential confounders (i.e. age, gender, BMI, education level and household income) into the model increased the percentage of variance explained in HEI score to $18.2 \%(P<0.0001)$. General food label use $(\beta=1.18$ (se $0 \cdot 26), P<0 \cdot 0001)$ remained the only significant variable among the main eating behaviour traits and food label use variables and it explained $6.7 \%$ of the variance in HEI score, although a tendency was observed for TFEQ-cognitive restraint $\left(\beta=0 \cdot 28(\operatorname{se} 0 \cdot 15), \quad R^{2}=1 \cdot 2 \%\right.$, $P=0.06)$. Gender $(\beta=4.71$ (se 1.30), $P=0.0004)$ and BMI $(\beta=-0.32$ (sE 0.12$), P=0.01)$ explained respectively 4.3 and $2.2 \%$ of the variance in HEI score, indicating that women and those with a lower BMI had higher diet quality. Age and experimental conditions 1 and 2 remained in the model but were not significant $(P>0.05)$.

The model was also tested with the subscales that were significantly correlated with the HEI score. The first model thus included rigid and flexible control, unconditional permission to eat, general food label use, item seeking on food labels and experimental conditions. General food label use $(\beta=1.26$ (se 0.26), $P<0 \cdot 0001)$ and flexible control $(\beta=0.85$ (se 0.38$), P=0.03)$ respectively explained 6.9 and $1.5 \%$ of the variance in HEI score. The model explained $10.3 \%$ of the variance in HEI score $(P<0 \cdot 0001)$. In the fully adjusted model, the percentage of variance explained in HEI score increased to $17.5 \%(P<0.0001)$. Among the eating behaviour traits and food label use variables, general food label use $(\beta=1.09$ (SE 0.26), $P<0.0001$ ) remained again the only significant determinant of HEI score, explaining $5.7 \%$ of its variance. Gender $(\beta=4.88(\operatorname{se~} 1.31), P=0.0002)$ and

Table 4 Associations between eating behaviour traits and food label use among women and men involved in two previous experimental studies $^{(24,25)}$

\begin{tabular}{|c|c|c|c|c|c|c|c|c|}
\hline \multirow[b]{3}{*}{ Variable } & \multicolumn{4}{|c|}{ General food label use } & \multicolumn{4}{|c|}{ Item seeking on food labels } \\
\hline & \multicolumn{2}{|c|}{$\begin{array}{l}\text { Unadjusted } \\
\text { model }\end{array}$} & \multicolumn{2}{|c|}{$\begin{array}{l}\text { Fully adjusted } \\
\text { model }\end{array}$} & \multicolumn{2}{|c|}{$\begin{array}{l}\text { Unadjusted } \\
\text { model }\end{array}$} & \multicolumn{2}{|c|}{$\begin{array}{l}\text { Fully adjusted } \\
\text { model }\end{array}$} \\
\hline & $r$ & $P$ & $r$ & $P$ & $r$ & $P$ & $r$ & $P$ \\
\hline Cognitive restraint ${ }^{\star}$ & 0.37 & $<0.0001$ & 0.32 & $<0.0001$ & 0.26 & $<0.0001$ & 0.17 & 0.004 \\
\hline Rigid control & 0.35 & $<0.0001$ & 0.30 & $<0.0001$ & 0.22 & $<0.0001$ & 0.13 & 0.03 \\
\hline Flexible control & 0.33 & $<0.0001$ & 0.27 & $<0.0001$ & 0.22 & $<0.0001$ & 0.14 & 0.02 \\
\hline Disinhibition & 0.06 & 0.27 & 0.11 & 0.04 & 0.01 & 0.87 & 0.00 & 0.96 \\
\hline Susceptibility to hunger & 0.03 & 0.53 & 0.12 & 0.03 & -0.05 & 0.34 & 0.01 & 0.84 \\
\hline Restraint† & 0.19 & 0.0002 & 0.22 & $<0.0001$ & 0.06 & 0.24 & 0.01 & 0.84 \\
\hline Intuitive eating & -0.23 & $<0.0001$ & -0.24 & $<0.0001$ & -0.19 & 0.0004 & -0.17 & 0.003 \\
\hline Unconditional permission to eat & -0.35 & $<0.0001$ & -0.33 & $<0.0001$ & -0.33 & $<0.0001$ & -0.27 & $<0.0001$ \\
\hline Eating for physical rather than emotional reasons & -0.08 & 0.12 & -0.12 & 0.04 & -0.06 & 0.30 & -0.08 & 0.18 \\
\hline Reliance on internal hunger and satiety cues & 0.04 & 0.45 & 0.00 & 0.98 & 0.06 & 0.28 & 0.05 & 0.36 \\
\hline
\end{tabular}

$r$, partial Pearson's correlation coefficient.

Unadjusted model: adjusted only for experimental conditions.

Fully adjusted model: adjusted for experimental conditions, age, gender, BMI, education level and household income. Prefer not to answer for education level and household income were recoded as missing data. Education level and household income were treated as continuous variables. Five indicator variables were created for experimental conditions and the control group of Carbonneau et al.'s study ${ }^{(24)}$ was used as reference.

General food label use: unadjusted model, $n 359$ to 379 ; fully adjusted model, $n 323$ to 339 .

Item seeking on food labels: unadjusted model, $n 333$ to 346 ; fully adjusted model, $n 302$ to 314 .

*Assessed with the Three-Factor Eating Questionnaire ${ }^{(1,29)}$.

†Assessed with the Restraint Scale ${ }^{(30)}$. 

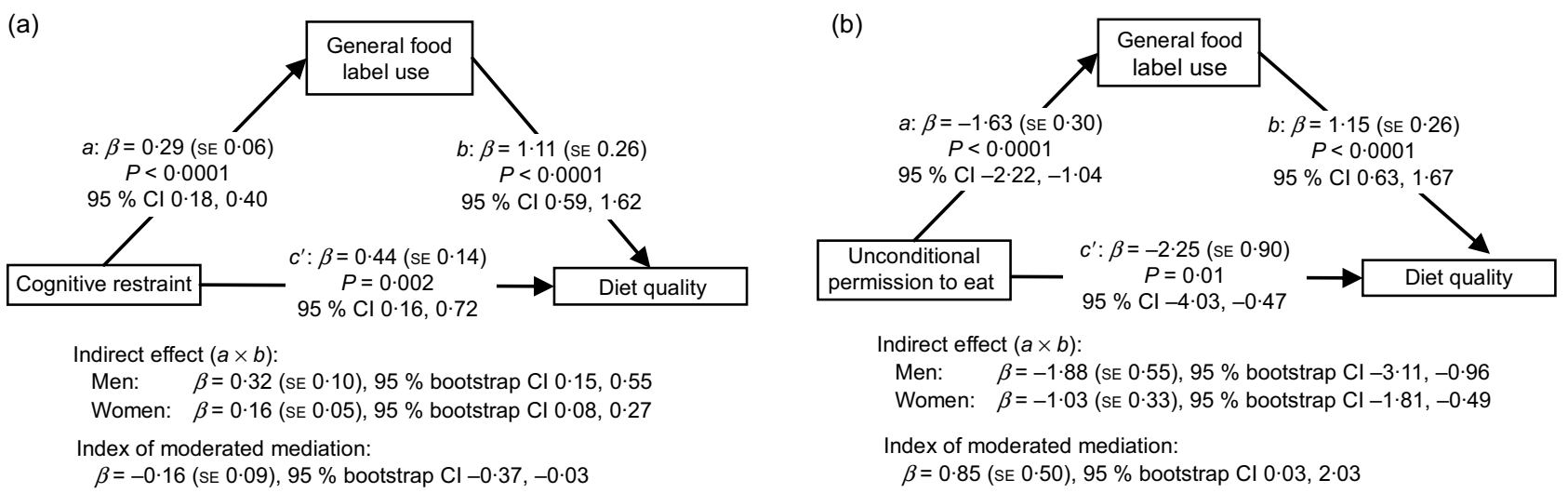

Fig. 1 Mediating effect of general food label use on the association between cognitive restraint (a) or unconditional permission to eat (b) and diet quality among men and women involved in two previous experimental studies ${ }^{(24,25)}$. $a=\beta$ coefficient for the association between cognitive restraint (a) or unconditional permission to eat (b) and general food label use. $b=\beta$ coefficient for the association between general food label use and diet quality (HEI score). $c^{\prime}=\beta$ coefficient for the association between cognitive restraint (a) or unconditional permission to eat (b) and diet quality (HEl score) when the mediator (general food label use) is in the model. Data obtained from Process model 7 for (a) and (b). Models adjusted for experimental conditions, age, BMl, education level and household income. Prefer not to answer for education level and household income were recoded as missing data. Education level and household income were treated as continuous variables. Five indicator variables were created for experimental conditions and the control group of Carbonneau et al.'s study(24) was used as reference. Cognitive restraint was assessed with the Three-Factor Eating Questionnaire ${ }^{(1,29)}$. (a) $n 329 ;$ (b) $n 336$

BMI $(\beta=-0.42(\operatorname{se} 0 \cdot 12), P=0.0006)$ explained 4.6 and $4.0 \%$ in the variance in HEI score, respectively, again suggesting that women and individuals with a lower BMI had better diet quality. Unconditional permission to eat and experimental conditions 1 and 2 remained in the model but did not significantly contribute to explain the HEI score $(P>0.05)$.

Additional analyses were performed to test whether food label use variables could mediate the association between eating behaviour traits (i.e. cognitive restraint and its two subscales, and unconditional permission to eat) and diet quality since these eating behaviour traits were associated with food label use variables and diet quality (Fig. 1, Table 5). Results showed that general food label use was a partial mediator of the associations between TFEQcognitive restraint, flexible control and unconditional permission to eat and HEI score and that general food label use mediated the association between rigid control and HEI score. Moreover, the index of moderated mediation indicated that the mediating effects were stronger in men than in women, except for the model with rigid control since the index of moderated mediation did not reach significance ( $95 \%$ bootstrap CI $-1 \cdot 46,0 \cdot 02$ ). A similar pattern of association was observed in the models testing the mediating effect of item seeking on food labels. Indeed, item seeking on food labels partially mediated the association of TFEQcognitive restraint and rigid control with HEI score, but this was observed only in men. Item seeking on food labels partially mediated the association between flexible control and HEI score with no moderating effect of gender. Finally, item seeking on food labels partially mediated the association between unconditional permission to eat and HEI score and the mediating effect was stronger in men than in women (Table 5).

\section{Discussion}

The present study aimed to assess the associations among eating behaviour traits, food label use and diet quality and to examine whether the association between eating behaviour traits and diet quality was mediated by food label use. Among the variables examined in the study, general food label use appears to be the main determinant of diet quality although correlation analyses also showed positive associations between TFEQ-cognitive restraint, and its subscales, and diet quality (HEI score) and negative associations of intuitive eating (in the model that was adjusted only for experimental condition), and its subscale unconditional permission to eat, with diet quality. Results also revealed that the associations between most of these eating behaviour traits and diet quality were partially mediated by general food label use and item seeking on food labels and the mediating effect was stronger in men than in women in most models.

The pattern of associations between eating behaviour traits and diet quality is consistent with the literature. Indeed, the positive association between cognitive restraint and diet quality has been previously reported by studies showing that cognitive restraint and flexible control were associated with higher intakes of foods that are components of healthy eating such as green vegetables, fish and yoghurts or with a higher diet quality score based on fruit and vegetables, wholegrain products and fish intakes, respectively ${ }^{(4,5)}$. The positive association between rigid control and diet quality must however be interpreted with caution since restrained eaters may be more prone to social desirability bias when reporting eating habits ${ }^{(35)}$. Because rigid control is characterized by a dichotomous (all or nothing) approach towards eating and has been positively 


\section{Public Health Nutrition}

Table 5 Mediation models between eating behaviour traits, food label use and diet quality (HEI score) among women and men involved in two previous experimental studies ${ }^{(24,25)}$

\begin{tabular}{|c|c|c|c|c|c|c|c|c|c|c|c|c|c|c|c|c|c|c|c|c|}
\hline & \multicolumn{4}{|c|}{$a^{*}$} & \multicolumn{4}{|c|}{$b \dagger$} & \multicolumn{4}{|c|}{ Direct effect $\left(c^{\prime}\right) \ddagger$} & \multicolumn{4}{|c|}{ Indirect effect $(a \times b)$} & \multicolumn{3}{|c|}{$\begin{array}{l}\text { Index of moderated } \\
\text { mediation }\end{array}$} & \multirow[b]{2}{*}{$\begin{array}{c}\text { Process } \\
\text { model } \\
\text { used }\end{array}$} \\
\hline & $\beta$ & SE & $P$ & $95 \% \mathrm{Cl}$ & $\beta$ & SE & $P$ & $95 \% \mathrm{Cl}$ & $\beta$ & SE & $P$ & $95 \% \mathrm{Cl}$ & & $\beta$ & SE & $\begin{array}{c}95 \% \\
\text { bootstrap } \\
\mathrm{Cl}\end{array}$ & $\beta$ & SE & $\begin{array}{c}95 \% \\
\text { bootstrap } \\
\mathrm{Cl}\end{array}$ & \\
\hline \multicolumn{21}{|c|}{ Mediator: General food label use } \\
\hline Rigid control§ & 0.45 & 0.08 & $<0.0001$ & $0.29,0.62$ & $1 \cdot 14$ & 0.26 & $<0.0001$ & $0.63,1.65$ & 0.37 & 0.39 & 0.35 & $-0.41,1.15$ & & 0.52 & 0.14 & $0.28,0.83$ & -0.63 & 0.38 & $-1.46,0.02$ & $4 \pi$ \\
\hline Flexible control & 0.61 & $0 \cdot 14$ & $<0.0001$ & $0.33,0.89$ & $1 \cdot 21$ & 0.26 & $<0.0001$ & $0 \cdot 70,1 \cdot 73$ & 0.90 & 0.37 & 0.01 & $0.18,1.62$ & $\begin{array}{l}\text { Men } \\
\text { Women }\end{array}$ & $\begin{array}{l}0.74 \\
0.36\end{array}$ & $\begin{array}{l}0.23 \\
0.13\end{array}$ & $\begin{array}{l}0.37,1.30 \\
0.15,0.64\end{array}$ & -0.39 & 0.23 & $-0.93,-0.03$ & 7 \\
\hline \multicolumn{21}{|c|}{ Mediator: Item seeking on food labels } \\
\hline Cognitive restraint $\|$ & 0.35 & 0.09 & 0.0002 & $0.16,0.53$ & 0.59 & 0.16 & 0.0004 & $0.27,0.92$ & 0.53 & 0.14 & 0.0003 & $0.24,0.81$ & $\begin{array}{l}\text { Men } \\
\text { Women }\end{array}$ & $\begin{array}{l}0.21 \\
0.04\end{array}$ & $\begin{array}{l}0.10 \\
0.03\end{array}$ & $\begin{array}{r}0.06,0.44 \\
-0.02,0.12\end{array}$ & -0.17 & 0.09 & $-0.40,-0.02$ & 7 \\
\hline Rigid control & 0.90 & 0.27 & 0.001 & $0.37,1.43$ & 0.61 & 0.17 & 0.0004 & $0.27,0.94$ & 1.07 & 0.39 & 0.007 & $0 \cdot 30,1 \cdot 85$ & $\begin{array}{l}\text { Men } \\
\text { Women }\end{array}$ & $\begin{array}{l}0.54 \\
0.07\end{array}$ & $\begin{array}{l}0.26 \\
0.09\end{array}$ & $\begin{array}{r}0.14,1.18 \\
-0.08,0.28\end{array}$ & -0.48 & 0.27 & $-1.11,-0.06$ & 7 \\
\hline Flexible control§ & 0.31 & 0.13 & 0.02 & $0.05,0.58$ & 0.52 & $0 \cdot 16$ & 0.002 & $0.20,0.85$ & 0.75 & 0.38 & 0.047 & $0.01,1.49$ & & 0.16 & 0.09 & $0.03,0.40$ & -0.29 & 0.23 & $-0.86,0.08$ & $4^{\star \star}$ \\
\hline $\begin{array}{l}\text { Unconditional } \\
\text { permission to eat }\end{array}$ & -2.54 & 0.50 & $<0.0001$ & $-3.52,-1.56$ & 0.50 & 0.17 & 0.003 & $0.17,0.83$ & $-2 \cdot 81$ & 0.94 & 0.003 & $-4.67,-0.96$ & $\begin{array}{l}\text { Men } \\
\text { Women }\end{array}$ & $\begin{array}{l}-1 \cdot 27 \\
-0.45\end{array}$ & $\begin{array}{l}0.50 \\
0.26\end{array}$ & $\begin{array}{l}-2.51,-0.46 \\
-1 \cdot 16,-0.08\end{array}$ & 0.82 & 0.44 & $0.14,1.96$ & 7 \\
\hline
\end{tabular}

HEI, Canadian Healthy Eating Index (28).

Models using general food label use as a mediator, $n 323$ to 325 ; models using item seeking on food labels as a mediator, $n 302$ to 312 .

${ }^{*} a=\beta$ coefficient for the association between eating behaviour traits and general food label use/item seeking on food labels.

$+b=\beta$ coefficient for the association between general food label use/item seeking on food labels and diet quality (HEI score).

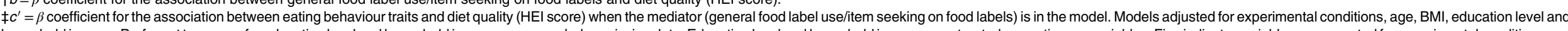

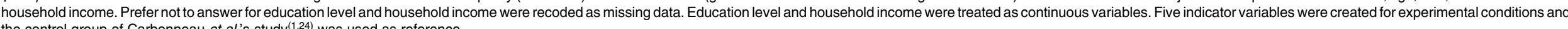
the control group of Carbonneau et al.'s study (1,24) was used as reference.

$\S$ This model was also adjusted for gender as gender did not moderate the mediation effect.

$\|$ Assessed with the Three-Factor Eating Questionnaire (1,29) $^{2}$

IIndex of moderated mediation was obtained from Process model 58, but it was also non-significant in other moderated mediation models (i.e. models 14 and 7 ).

${ }^{*}$ Index of moderated mediation was obtained from Process model 7, but it was also non-significant in other moderated mediation models (i.e. models 58 and 14). 
associated with disinhibition ${ }^{(13-15)}$, it may not be a positive determinant of diet quality in the longer term. Accordingly, it has been suggested that a high level of cognitive restraint in women may be difficult to sustain over time ${ }^{(14)}$. In contrast to rigid control, flexible control represents an approach towards eating that is characterized by a higher probability of successful weight reduction or weight management and by a negative association with disinhibition ${ }^{(14,15)}$. Such literature suggests that flexible control may be easier to maintain over time, so individuals may be less likely to show important deviations from their usual dietary habits and thus they may eat less unhealthy foods as supported by a positive association with diet quality $^{(5)}$. Although the negative association between unconditional permission to eat and diet quality has not been previously reported, this intuitive eating subscale has been associated with lower fruit and vegetable and wholegrain intakes ${ }^{(39)}$, which represent important components of diet quality.

Food label use, as measured by both general food label use and item seeking on food labels, was associated with a better diet quality. This is in line with the majority of studies on this topic demonstrating that food label use benefits eating habits ${ }^{(20)}$. Considering the cross-sectional nature of the present study, it is also possible that individuals having a better diet quality pay more attention to food labels, as it has been reported that individuals having better eating habits report a greater use of food labels ${ }^{(20)}$. To our knowledge, the present study is one of the first to document the associations between eating behaviour traits and food label use. The positive associations among restrained eating and food label use were expected since restrained eating requires cognitive effort to adhere to a diet in order to lose or maintain body weight, and nutritional information found on food labels could support that effort. Similarly, the negative association between intuitive eating and food label use suggests that because intuitive eating relies on internal sensations of hunger and satiety, it could be less related to cognitive processes towards eating such as using food labels when purchasing or consuming food. Disinhibition and susceptibility to hunger were both positively, but weakly, associated with general food label use in the fully adjusted model, suggesting that individuals presenting a higher level of disinhibition or susceptibility to hunger may use food labels to select food products that seem healthier or lower in fat or in energy to compensate for their overeating tendencies. Likewise, individuals with susceptibility to hunger may also use food labels to choose foods that seem more satiating, but it is also possible that choosing low-fat or low-energy foods triggers hunger sensations.

Although eating behaviour traits and food label use explained only a small proportion of the variance in diet quality, the present study suggests that food label use greater explains diet quality than eating behaviour traits.
Given the myriad of factors influencing dietary habits ${ }^{(40)}$, the percentage of variance in diet quality explained by the different models, and mainly by food label use, is nevertheless considerable. Moreover, using food labels seems to represent a tool that explained a greater part of the association between cognitive restraint and diet quality in men than in women. In contrast, not using food labels seems to greater explain the negative association between unconditional permission to eat and diet quality in men compared with women. Considering that men are more prone to give themselves unconditional permission to eat compared with women $^{(39)}$, this latter result suggests that when men allow themselves to eat unconditionally, they use food labels less often, which contributes to a lower diet quality. This result is in line with Christoph et al.'s study showing that a higher level of intuitive eating was associated with a lower level of food label use in men ${ }^{(23)}$. The fact that women usually eat less intuitively ${ }^{(11,16,17)}$, are more restrained ${ }^{(13-15)}$ and use food labels more often than men ${ }^{(20,21)}$ may explain why the mediating effect of food label use was weaker in women. Similarly, restrained eating and dieting represent a cultural norm for women in Western countries ${ }^{(41,42)}$, suggesting that women may more importantly internalize these behaviours. This context might explain why the association between cognitive restraint and diet quality is less mediated by food label use in women as opposed to restrained men, who more essentially need to rely on such tool to achieve a better diet quality.

Nevertheless, and irrespective of gender, the mediating effect of food label use in the association between cognitive restraint and diet quality is in line with the use of dietary restraint as a self-regulation strategy as proposed by Schaumberg et al. ${ }^{(43)}$. Accordingly, our results suggest that food labelling could support self-monitoring among restrained eaters, helping them to implement their dieting rules and reach a better diet quality, which is in line with a previous study showing that the association between attitude towards healthy meal preparation and diet quality was mediated by a greater use of food labels among college students ${ }^{(44)}$. Christoph et al. also showed that food label use was associated with a greater likelihood of engaging in healthy weight-control behaviours, but also, and to a lesser extent, to a greater likelihood of engaging in unhealthy weight-control behaviours ${ }^{(23)}$, suggesting that some individuals may use food labels to implement unfavourable eating behaviours. It is important to note that the associations observed in the present study are not causal due to its cross-sectional nature.

The present study has several strengths and limitations that need to be outlined. First, it is the first study to assess the associations of many eating behaviour traits with food label use and global diet quality in the same sample of men and women, and to our knowledge, the mediating effect of food label use in the association between these eating behaviour traits and diet quality has never been 
explored. As previously discussed, the main limitation of the study is its cross-sectional nature that does not allow to determine causality among variables. Therefore, it is not possible to confirm if restrained individuals use food labels because of their dieting behaviours or if using food labels when making food choices can lead to restrained eating. Moreover, because of the rather conservative strategy used regarding mediation analyses, it is possible that other mediating effects could have been observed for eating behaviour traits that were not directly associated with diet quality (HEI score). However, this was beyond the scope of the present study and therefore remains to be investigated. Dietary intake was self-reported, implying that potential social desirability bias could have influenced the results. The use of an FFQ may be implicated in the small proportion of the variance in diet quality explained by eating behaviour traits and food label use variables. While the Cronbach's $\alpha$ coefficient of one of the TFEQ subscales is rather low (i.e. $<0.60$ ) and could be considered as a limitation, the TFEQ remains an established questionnaire used to measure eating behaviour traits and the Cronbach's $\alpha$ coefficients of its three main components were adequate in this sample. With regard to the low Cronbach's $\alpha$ coefficient for general food label use (i.e. 0.57), the validity of the Label Reading Survey has been previously reported ${ }^{(32)}$ and, as mentioned earlier, the Cronbach's $\alpha$ coefficient for our adapted questionnaire was similar to the value of the original one. It is also likely that the different contexts specified in the questions (i.e. using food labels when purchasing a food for the first time or when eating food) are implicated in this relatively low internal consistency for the general food label use variable, since one could use food labels only at the point of purchase. The Label Reading Survey covered the main food label components (i.e. nutrition facts table, health and nutrient-related claims), but did not cover all information included on food labels (e.g. ingredients list and other types of claims), and this may reduce the accuracy of the food label use measure. The high education level of participants could limit the generalization of the results to other populations. Finally, it is important to mention that this cross-sectional study was conducted among participants of two previous studies. One could argue that this design implicated priming which can impact the results of the present study. However, as previously mentioned, no experimental conditions effect was observed for the main eating behaviour traits, food label use variables or measured energy intake in the main studies (i.e. ad libitum snack test or $10 \mathrm{~d}$ energy intake). Moreover, this potential priming effect was considered in all analyses by adding the experimental conditions of the main studies as a covariate, so it is likely that the results observed in the present study are not explained by the priming effect of the main studies or that this bias is therefore greatly reduced.

\section{Conclusions}

The present study showed that food label use was a better determinant of diet quality than eating behaviour traits. Moreover, food label use partially mediated the association between cognitive restraint or unconditional permission to eat and diet quality and the mediating effects were stronger in men than in women. While food labels could be helpful to adopt a healthy diet, the psychobehavioural profile of individuals seen in a clinical context should be assessed to individualize strategies used to facilitate healthy eating. It is indeed important to support appropriate self-regulation strategies and not favour the adoption of unhealthy eating behaviour traits that may result in counter-regulatory eating ${ }^{(43)}$. Future studies should assess the impact of food label use on eating behaviour traits and diet quality in an intervention context.

\section{Acknowledgements}

Acknowledgements: The authors express their gratitude to the participants for their involvement in the two studies as well as to the members of the research team who helped conduct the studies. The authors also thank Shirin Panahi for editing the manuscript. Financial support: Data collection that allowed the present study was supported by grants from the Canadian Institutes of Health Research (CIHR; grant numbers MOP-110951 and OOP-94065) and the Danone Institute. R.J. is the recipient of a PhD scholarship from the Fonds de recherche du Québec - Santé (FRQS). CIHR and Danone Institute had no role in the design, analysis or writing of this article. Conflict of interest: None. Authorship: R.J., V.D. and V.P. conceived the study and its design. S.P. coordinated data collection. R.J. performed statistical analyses and wrote the first draft of the manuscript. R.J., V.D., B.L., E.D., S.P. and V.P. participated in data interpretation, critically reviewed the manuscript and approved the final version. Ethics of buman subject participation: This study was conducted according to the guidelines laid down in the Declaration of Helsinki and all procedures (of both studies) involving human subjects were approved by the Laval University ethics committee. Written informed consent was obtained from all subjects.

\section{References}

1. Stunkard AJ \& Messick S (1985) The three-factor eating questionnaire to measure dietary restraint, disinhibition and hunger. J Psychosom Res 29, 71-83.

2. Bryant EJ, King NA \& Blundell JE (2008) Disinhibition: its effects on appetite and weight regulation. Obes Rev 9, 409-419.

3. Fung TT, Pan A, Hou T et al. (2015) Long-term change in diet quality is associated with body weight change in men and women. J Nutr 145, 1850-1856. 
4. de Lauzon B, Romon M, Deschamps V et al. (2004) The Three-Factor Eating Questionnaire-R18 is able to distinguish among different eating patterns in a general population. J Nutr 134, 2372-2380.

5. Swan E, Bouwman L, Hiddink GJ et al. (2015) Profiling healthy eaters. Determining factors that predict healthy eating practices among Dutch adults. Appetite 89, 122-130.

6. Dykes J, Brunner EJ, Martikainen PT et al. (2004) Socioeconomic gradient in body size and obesity among women: the role of dietary restraint, disinhibition and hunger in the Whitehall II study. Int J Obes Relat Metab Disord 28, 262-268.

7. Bellisle F, Clement K, Le Barzic M et al. (2004) The Eating Inventory and body adiposity from leanness to massive obesity: a study of 2509 adults. Obes Res 12, 2023-2030.

8. French SA, Mitchell NR, Wolfson J et al. (2014) Questionnaire and laboratory measures of eating behavior. Associations with energy intake and BMI in a community sample of working adults. Appetite 72, 50-58.

9. Tribole E \& Resch E (2012) Intuitive Eating - A Revolutionary Program that Works. New York: St. Martin's Press.

10. Madden CE, Leong SL, Gray A et al. (2012) Eating in response to hunger and satiety signals is related to BMI in a nationwide sample of 1601 mid-age New Zealand women. Public Health Nutr 15, 2272-2279.

11. Smith $\mathrm{T} \&$ Hawks SR (2006) Intuitive eating, diet composition, and the meaning of food in healthy weight promotion. Am J Health Educ 37, 130-136.

12. Van Dyke N \& Drinkwater EJ (2014) Relationships between intuitive eating and health indicators: literature review. Public Health Nutr 17, 1757-1766.

13. Provencher V, Drapeau V, Tremblay A et al. (2003) Eating behaviors and indexes of body composition in men and women from the Quebec family study. Obes Res $\mathbf{1 1}$, 783-792.

14. Drapeau V, Provencher V, Lemieux S et al. (2003) Do 6-y changes in eating behaviors predict changes in body weight? Results from the Quebec Family Study. Int J Obes Relat Metab Disord 27, 808-814.

15. Westenhoefer J, Stunkard AJ \& Pudel V (1999) Validation of the flexible and rigid control dimensions of dietary restraint. Int J Eat Disord 26, 53-64.

16. Denny KN, Loth K, Eisenberg ME et al. (2013) Intuitive eating in young adults. Who is doing it, and how is it related to disordered eating behaviors? Appetite 60, 13-19.

17. Tylka TL \& Kroon Van Diest AM (2013) The Intuitive Eating Scale-2: item refinement and psychometric evaluation with college women and men. J Couns Psychol 60, 137-153.

18. Cowburn G \& Stockley L (2005) Consumer understanding and use of nutrition labelling: a systematic review. Public Health Nutr 8, 21-28.

19. Cha E, Kim KH, Lerner HM et al. (2014) Health literacy, selfefficacy, food label use, and diet in young adults. Am J Health Behav 38, 331-339.

20. Campos S, Doxey J \& Hammond D (2011) Nutrition labels on pre-packaged foods: a systematic review. Public Health Nutr 14, 1496-506.

21. Christoph MJ, Larson N, Laska MN et al. (2018) Nutrition facts panels: who uses them, what do they use, and how does use relate to dietary intake? J Acad Nutr Diet 118, 217-228.

22. Provencher V \& Jacob R (2016) Impact of perceived healthiness of food on food choices and intake. Curr Obes Rep 5, 65-71.

23. Christoph MJ, Loth KA, Eisenberg ME et al. (2018) Nutrition facts use in relation to eating behaviors and healthy and unhealthy weight control behaviors. J Nutr Educ Behav 50, 267-274.e1.

24. Carbonneau E, Perron J, Drapeau V et al. (2015) Impact of nutritional labelling on 10-d energy intake, appetite perceptions and attitudes towards food. Br J Nutr 114, 2138-2147.
25. Gravel K, Doucet E, Herman CP et al. (2012) 'Healthy,' 'diet,' or 'hedonic'. How nutrition claims affect food-related perceptions and intake? Appetite 59, 877-884.

26. Goulet J, Nadeau G, Lapointe A et al. (2004) Validity and reproducibility of an interviewer-administered food frequency questionnaire for healthy French-Canadian men and women. Nutr J 3, 13.

27. Labonte ME, Cyr A, Baril-Gravel L et al. (2012) Validity and reproducibility of a web-based, self-administered food frequency questionnaire. Eur J Clin Nutr 66, 166-173.

28. Dubois L, Girard M \& Bergeron N (2000) The choice of a diet quality indicator to evaluate the nutritional health of populations. Public Health Nutr 3, 357-365.

29. Llunch A (1995) Identification des conduites alimentaires par approches nutritionnelles et psychomériques: implications thérapeutiques et prévention dans l'obésité humaine (Identification of food intake behaviors by nutritional and psychometric means: implications for prevention and treatment of human obesity). PhD Thesis, Université Henri Poincaré.

30. Herman CP \& Mack D (1975) Restrained and unrestrained eating. J Pers 43, 647-660.

31. Tylka TL (2006) Development and psychometric evaluation of a measure of intuitive eating. J Couns Psychol 53, 226-240.

32. Marietta AB, Welshimer KJ \& Anderson SL (1999) Knowledge, attitudes, and behaviors of college students regarding the 1990 Nutrition Labeling Education Act food labels. J Am Diet Assoc 99, 445-449.

33. Johansson L, Solvoll K, Bjorneboe GE et al. (1998) Underand overreporting of energy intake related to weight status and lifestyle in a nationwide sample. Am J Clin Nutr $\mathbf{6 8}$, 266-274.

34. Goldberg GR, Black AE, Jebb SA et al. (1991) Critical evaluation of energy intake data using fundamental principles of energy physiology: 1. Derivation of cut-off limits to identify under-recording. Eur J Clin Nutr 45, 569-581.

35. Asbeck I, Mast M, Bierwag A et al. (2002) Severe underreporting of energy intake in normal weight subjects: use of an appropriate standard and relation to restrained eating. Public Health Nutr 5, 683-690.

36. Hayes AF (2013) Introduction to Mediation, Moderation and Conditional Process Analysis: A Regression-Based Approach. New York: The Guilford Press.

37. Perneger TV (1998) What's wrong with Bonferroni adjustments. BMJ 316, 1236-1238.

38. Baron RM \& Kenny DA (1986) The moderator-mediator variable distinction in social psychological research: conceptual, strategic, and statistical considerations. J Pers Soc Psychol 51, 1173-1182.

39. Camilleri GM, Mejean C, Bellisle F et al. (2017) Intuitive eating dimensions were differently associated with food intake in the general population-based NutriNet-Santé study. J Nutr 147, 61-69.

40. Sleddens EF, Kroeze W, Kohl LF et al. (2015) Correlates of dietary behavior in adults: an umbrella review. Nutr Rev 73, 477-499.

41. Rolls BJ, Fedoroff IC \& Guthrie JF (1991) Gender differences in eating behavior and body weight regulation. Health Psychol 10, 133-142.

42. Vartanian LR, Herman CP \& Polivy J (2007) Consumption stereotypes and impression management: how you are what you eat. Appetite 48, 265-277.

43. Schaumberg K, Anderson DA, Anderson LM et al. (2016) Dietary restraint: what's the harm? A review of the relationship between dietary restraint, weight trajectory and the development of eating pathology. Clin Obes 6, 89-100.

44. Graham DJ \& Laska MN (2012) Nutrition label use partially mediates the relationship between attitude toward healthy eating and overall dietary quality among college students. J Acad Nutr Diet 112, 414-418. 